\title{
Spatio-temporal statistics: applications in epidemiology, veterinary medicine and ecology
}

\author{
Annibale Biggeri, ${ }^{1}$ Dolores Catelan, ${ }^{1}$ David Conesa, ${ }^{2}$ Penelope Vounatsou ${ }^{3}$ \\ ${ }^{1}$ Department of Statistics, Computer Science, Applications, University of Florence, \\ Florence, Italy; ${ }^{2}$ Department of Statistics and Operational Research, University of \\ Valencia, Valencia, Spain; ${ }^{3}$ Department of Epidemiology and Public Health, Swiss Tropical \\ and Public Health Institute, Basel, Switzerland
}

This special issue presents the papers given at the satellite conference of the XXVII International Biometric Conference held in Valencia on July $13^{\text {th }}-14^{\text {th }}, 2014$ and organised by the Spanish Region of the International Biometric Society. The theme and main topic of the satellite conference was spatio-temporal statistics with particular emphasis on applications in epidemiology, veterinary medicine, ecology and life sciences.

The issue contains seven original contributions and one short communication. Three papers addressed applications in human spatial epidemiology. Boscoe et al. (2016) presented a spatial analysis on a public domain data set on cancer incidence at the census block level. As the authors stated there has long been a demand for Ipublicly available] cancer incidence data at fine geographic resolutions with data enrichment needed on race, ethnicity and socioeconomic indicators. In an open data era the paper demonstrates the potentiality and usefulness of such information over a wide range of spatial analyses. Cecconi et al. (2016b) disentangled the spatial distribution of cutaneous melanoma in an Italian region by applying a bi-variate analysis on melanoma and lip cancer incidence data. The application was felt

Correspondence: Dolores Catelan, Department of Statistics, Computer Science, Applications "G. Parenti", University of Florence, viale Giovan Battista Morgagni 59, 50134 Florence, Italy.

Tel: +39.055 .275 .1500 .

E-mail: catelan@disia.unifi.it

Key words: Preferential sampling; Veterinary parasitological surveillance; Livestock.

Acknowledgements: the research leading to these results has received funding from the European Union Seventh Framework Programme FP7-KBBE2011-5 under grant agreement no 288975 and University of Florence, ex $60 \%$.

Received for publication: 22 February 2016.

Accepted for publication: 26 February 2016.

(C) Copyright A. Biggeri et al., 2016

Licensee PAGEPress, Italy

Geospatial Health 2016; 11:469

doi:10.4081/gh.2016.469

This article is distributed under the terms of the Creative Commons Attribution Noncommercial License (CC BY-NC 4.0) which permits any noncommercial use, distribution, and reproduction in any medium, provided the original author(s) and source are credited. to be original and useful as it highlights the particular features of the spatial pattern with findings suggesting diagnostic/preventive interventions to be instituted. Fazzo et al. (2016) focused on a priori indentified high-risk areas for environmental pollution following the Seveso directive. Their paper referred to an analysis of the spatial distribution of cancer incidence within the high-risk area of Priolo (Sicily, Italy) with the discussion centered on how to estimate the impact of industrial pollution on population health.

Two papers were presented in the veterinary spatial epidemiology context, both focusing on Fasciola hepatica infection - a severe parasitological disease, which has a strong impact on animal health with sizable economic losses as a consequence. Barber et al. (2016) modelled the spatial incidence of diseases using local environmental factors. The authors specify a hierarchical Bayesian spatial model that tackle the misalignment problem and measurement error on the covariates. Cecconi et al. (2016a) discussed the spatial analysis of parasitological data when collected by opportunistic surveys or informative sampling designs. A geostatistical model aimed at predicting the continuous and spatially-varying risk of a parasite infection was proposed, and a two-stage hierarchical Bayesian model specified that can be adjusted for preferential sampling. This latter issue was also discussed in a paper on assessment of exposure to air pollution (Grisotto et al., 2016). Using air quality data from a network of monitoring stations, a Bayesian approach was applied to account for prediction uncertainty and to integrate information provided by deterministic models based on emissions meteorology and chemico-physical characteristics of the atmosphere. The paper considered the situation in which the spatial process of interest and the sampling locations are not independent by a shared spatial random component model.

Spatial and temporal forecast of satellite time series data in the field of forest monitoring was addressed by Bayr et al. (2016). They employed several methods of functional data analysis, including functional principal component analysis, and a novel form of random regression forests with online learning capability. These approaches were applied to two study areas: in Germany to monitor forest damages caused by wind-storm, and in Spain to monitor forest fires.

Soil characteristics were modeled in the short communication by Neves et al. (2016). Classical geostatistical methods were used to analyse separately the spatial variability of each variable and to model the dependence structure of the data. The main objective of the study was to present some suggestions in order to give sustainable recommendations about the use of phosphorus fertilisers aiming at the establishment of pastures in these soils and thus support traditional livestock activity. 


\section{References}

Barber X, Conesa D, Lladosa S, López-Quílez A, 2016. Modelling the presence of disease under spatial misalignment using Bayesian latent Gaussian models. Geospat Health 11:415.

Bayr C, Gallaun H, Kleb U, Kornberger B, Steinegger M, Winter M, 2016. Satellite-based forest monitoring: spatial and temporal forecast of growing index and short wave infrared band. Geospat Health 11:310.

Boscoe FP, Talbot T0, Kulldorff M, 2016. Public domain small-area cancer incidence data for New York State, 2005-2009. Geospat Health 11:304.

Cecconi L, Biggeri A, Grisotto L, Berrocal V, Rinaldi L, Musella V, Cringoli G, Catelan D, 2016a. Preferential sampling in veterinary parasitological surveillance. Geospat Health 11:412.

Cecconi L, Busolin A, Barbone F, Serraino D, Chiarugi A, Biggeri A, Catelan D, 2016b. Spatial analysis of incidence of cutaneous melanoma in Friuli Venezia Giulia region in the period 1995-2005. Geospat Health 11:422.

Fazzo L, Carere M, Tisano F, Bruno C, Cernigliaro A, Cicero MR, Comba P, Contrino ML, De Santis M, Falleni F, Ingallinella V, Madeddu A, Marcello I, Regalbuto C, Sciacca G, Soggiu ME, Zona A, 2016. Cancer incidence in Priolo, Sicily: a spatial approach for estimation of industrial air pollution impact. Geospat Health 11:320.

Grisotto L, Consonni D, Cecconi L, Catelan D, Lagazio C, Bertazzi PA, Baccini M, Biggeri A, 2016. Geostatistical integration and uncertainty in pollutant concentration surface under preferential sampling. Geospat Health 11:426.

Odete Torres M, Neves MM, 2016. A preliminary spatial-temporal study of some soil characteristics in the calcareous massif of Sicó, Portugal. Geospat Health 11:342. 